\title{
DNA-Replikationsstress, Mitose und genomische Instabilität
}

\section{ALICIA KONRATH, ANN-KATHRIN SCHMIDT, HOLGER BASTIANS \\ INSTITUT FÜR MOLEKULARE ONKOLOGIE, SEKTION FÜR ZELLULÄRE ONKOLOGIE \\ UNIVERSITÄTSMEDIZIN GÖTTINGEN}

\section{Chromosomal instability (CIN) is a hallmark of cancer and contributes to tumorigenesis and tumor progression. While structural CIN (S-CIN) leads to structural chromosome aberrations, whole chromosome insta- bility (W-CIN) is defined by perpetual gains or losses of chromosomes during mitosis causing aneuploidy. Mitotic defects, but also abnormal DNA replication (replication stress) can lead to W-CIN. However, the functional link between replication stress, mitosis and aneuploidy is little understood.}

DOI: $10.1007 / \mathrm{s} 12268-021-1525-5$

(C) Die Autoren 2021

Eine chromosomale Instabilität (CIN) führt zu fortwährenden chromosomalen Veränderungen und kann in strukturelle CIN (S-CIN) und numerische CIN (W-CIN) unterteilt werden [1, 2]. S-CIN entsteht vor allem durch Defekte der DNA-Reparatur oder -Replikation und resultiert in strukturellen Chromosomenaberrationen, wie Deletionen oder Amplifikationen. W-CIN hingegen beschreibt die Fehlverteilung ganzer Chromosomen während der Mitose, was zur Induktion von Aneuploidie führt [2].
In Krebszellen spielen mitotische Defekte eine wichtige Rolle für die Erzeugung von W-CIN und Aneuploidie. Hier sind vor allem fehlerhafte, merotelische Kinetochor-Mikrotubuli-Anheftungen von besonderer Relevanz, bei denen ein Kinetochor eines Schwesterchromatids gleichzeitig mit Mikrotubuli beider Spindelpole verbunden ist. Diese fehlerhaften Mikrotubuli-Anheftungen führen typischerweise zu lagging chromosomes, der Vorstufe der Chromosomenfehlverteilung (Abb. 1A, [3]).
Eine Ursache für merotelische MikrotubuliAnheftungen sind überzählige Zentrosomen, die in der Mitose zu transienten multipolaren Spindel-Intermediaten führen und so die akkurate Chromatidentrennung stören (Abb. 1B, [4]). Überdies hat unser Labor eine erhöhte Wachstumsrate der Spindelmikrotubuli als einen krebsrelevanten Defekt identifiziert, der ebenfalls zur Erzeugung von lagging chromosomes in Krebszellen beiträgt (Abb. 1B). Eine Korrektur der abnormalen Mikrotubuli-Dynamik unterdrückt die Chromosomen-Fehlverteilungen, was den kausalen Zusammenhang zwischen abnormaler Mikrotubuli-Dynamik und Aneuploidie belegt [5]. Unser Labor konnte bereits wichtige Krebsgene identifizieren, die eine abnormale Mikrotubuli-Dynamik und W-CIN induzieren. Dazu gehören Tumorsuppressoren, wie BRCA1, CHK2 und p53/p73 [5-8]. Interessanterweise wurde aber auch gezeigt, dass DNA-Replikationsstress in der S-Phase zur Entstehung von W-CIN beitragen kann $[9,10]$.

\section{DNA-Replikation}

Die DNA-Replikation in der S-Phase ist ein Schlüsselereignis des Zellzyklus mit dem Ziel, das Genom basengenau zu duplizieren. Dabei entstehen zwei identische Schwester-

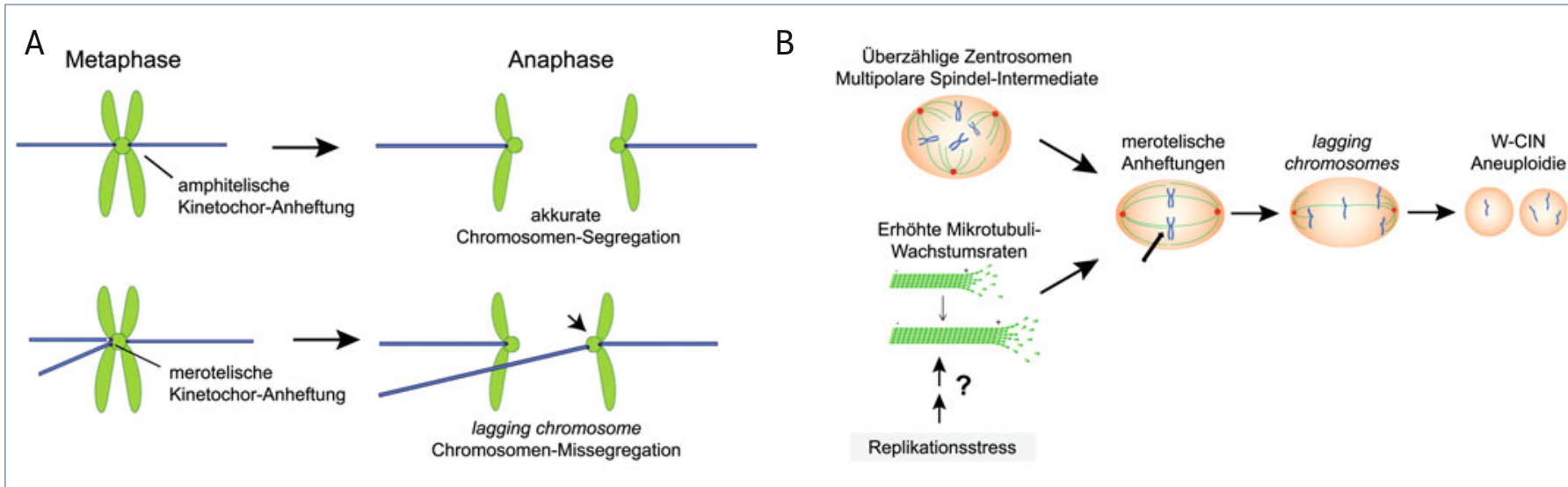

$\Delta$ Abb. 1: Ursachen für eine numerische chromosomale Instabilität (W-CIN). A, In Krebszellen können vermehrt fehlerhafte merotelische KinetochorMikrotubuli-Anheftungen auftreten, bei denen ein Kinetochor eines Schwesterchromatids gleichzeitig mit Mikrotubuli beider Spindelpole verbunden ist. Dies kann zur Erzeugung von lagging chromosomes, einer Vorstufe der Chromosomenfehlverteilung in der Anaphase, führen. B, Sowohl Hyper-Amplifikationen von Zentrosomen als auch erhöhte Wachstumsraten der Spindelmikrotubuli in der Mitose können die Ausbildung von lagging chromosomes bedingen und $\mathrm{W}-\mathrm{CIN}$ induzieren. 
chromatiden, die in der Mitose gerecht auf die Tochterzellen verteilt werden. Jegliche Störungen während der DNA-Replikation, die als DNA-Replikationsstress bezeichnet werden, können zu Veränderungen im Genom führen.

Der Start der Replikation ist ein zweischrittiger Prozess in der G1- und S-Phase des Zellzyklus (Abb. 2). In G1 werden PräReplikationskomplexe an den Stellen der Replikationsursprünge der DNA gebildet. Bei diesem licensing wird eine DNA-Bindestelle durch den ORC-Komplex (origin recognition complex) erkannt. Dieser rekrutiert unter Beteiligung der ATPase Cdc6 (cell division cycle 6) sowie Cdt1 (Cdc10dependent transcript 1) den Helikase-Komplex MCM (mini-chromosome maintenance) mit den Untereinheiten MCM2-7. Das licensing läuft an sämtlichen Replikationsursprüngen im Genom ab und ist regulatorisch von der anschließenden Aktivierung getrennt [11].

Die Aktivierung der Prä-Replikationskomplexe findet am Beginn der S-Phase statt. Die Kinasen Cdc7/DDK (DBF4dependent kinase) und CDK1/2 (cyclindependent kinase 1/2) phosphorylieren einige Replikationsfaktoren, wie MCM, CDC45, GINS und die DNA-Polymerase $\varepsilon$. Zudem initiieren CDKs und DDK in Anwesenheit von RFC (replication factor C), RPA (replication protein A) sowie PCNA (proliferating cell nuclear antigen) die Aktivierung der MCM-Helikase, was zur Entwindung der DNA und zur Bildung der Replikationsgabel führt. Die DNA-Synthese wird dann durch den Polymerase- $\alpha$-PrimaseKomplex gestartet. Leit- und Folgestrang werden anschließend durch die Polymerasen $\delta$ bzw. $\varepsilon$ verlängert (Abb. 2). In jedem Zellzyklus wird nur ein kleiner Anteil der Replikationsursprünge aktiviert, während die meisten Ursprünge dormant bleiben. Interessanterweise werden dormante Ursprünge bei Replikationsstress aktiviert, um eine Vervollständigung der Replikation zu gewährleisten [12, 13].

\section{Replikationsstress}

Unter Replikationsstress wird die Verlangsamung oder der Stopp der Replikation verstanden $[12,13]$. Replikationsstress führt in vielen Fällen zur Entstehung einzelsträngiger DNA (ssDNA), da die Helikase die DNA-Stränge weiter entwindet, während die DNA-Polymerase angehalten wird. Dabei wird einzelsträngige DNA durch RPA gebunden und stabilisiert, um ein Kollabieren der Replikationsgabel zu verhindern.

Häufige Ursachen für Replikationsstress sind DNA-Schäden, die den Fortschritt der Replikationsgabel behindern (Abb. 3A). Darüber hinaus können bestimmte DNA-Sequenzmotive Sekundärstrukturen ausbilden, welche die Replikation stören. Der fehlerhafte Einbau von Ribonukleotiden durch die weniger spezifischen Polymerasen $\varepsilon$ und $\delta$ kann ebenfalls Replikationsstress verursachen. Bei einem unkontrollierten Replikationsstart können zu viele Replikationsursprünge aktiviert werden, sodass erforderliche Komponenten schnell verbraucht werden und so die Replikation verlangsamen (Abb. 3B, C). Weiterhin können auch Kollisionen zwischen den Replikations- und Transkriptionsmaschinerien in der S-Phase zum Replikationsstress beitragen. Auch eine Rehybridisierung der mRNA mit der DNA unter Bildung eines 3fach-Strangs kann ursächlich für Replikationsstress sein (Abb. 3D).

\section{Zelluläre Antwort auf Replikations- stress}

Starker Replikationsstress aktiviert eine durch die ATR-Kinase (ataxia-telangectasia mutated, Rad3-related kinase) vermittelte Checkpoint-Antwort. Durch die Aktivierung von ATR wird der ZellzyklusFortschritt gehemmt und spät replizierende Replikationsursprünge werden unterdrückt, was Zeit für eine Reparatur gibt. Der ATR-Signalweg limitiert so S-CIN [12, 13].

Neben der ATR-vermittelten Checkpoint-Antwort löst Replikationsstress auch eine lokale Aktivierung einzelner (ansonsten dormanter) Replikationsursprünge aus, um die Replikation trotz Verlangsamung der Replikationsgabeln fertigzustellen. Die zugrunde liegenden Mechanismen sind bislang jedoch wenig verstanden.

Eine Konsequenz, insbesondere von mildem Replikationsstress, kann jedoch auch eine unvollständige DNA-Replikation sein. Tritt eine Zelle in Gegenwart von unvollständig replizierter DNA in die Mitose ein, wird eine akkurate Trennung der Schwesterchromatiden verhindert.
Hier steht eine Anzeige.

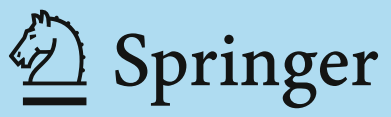




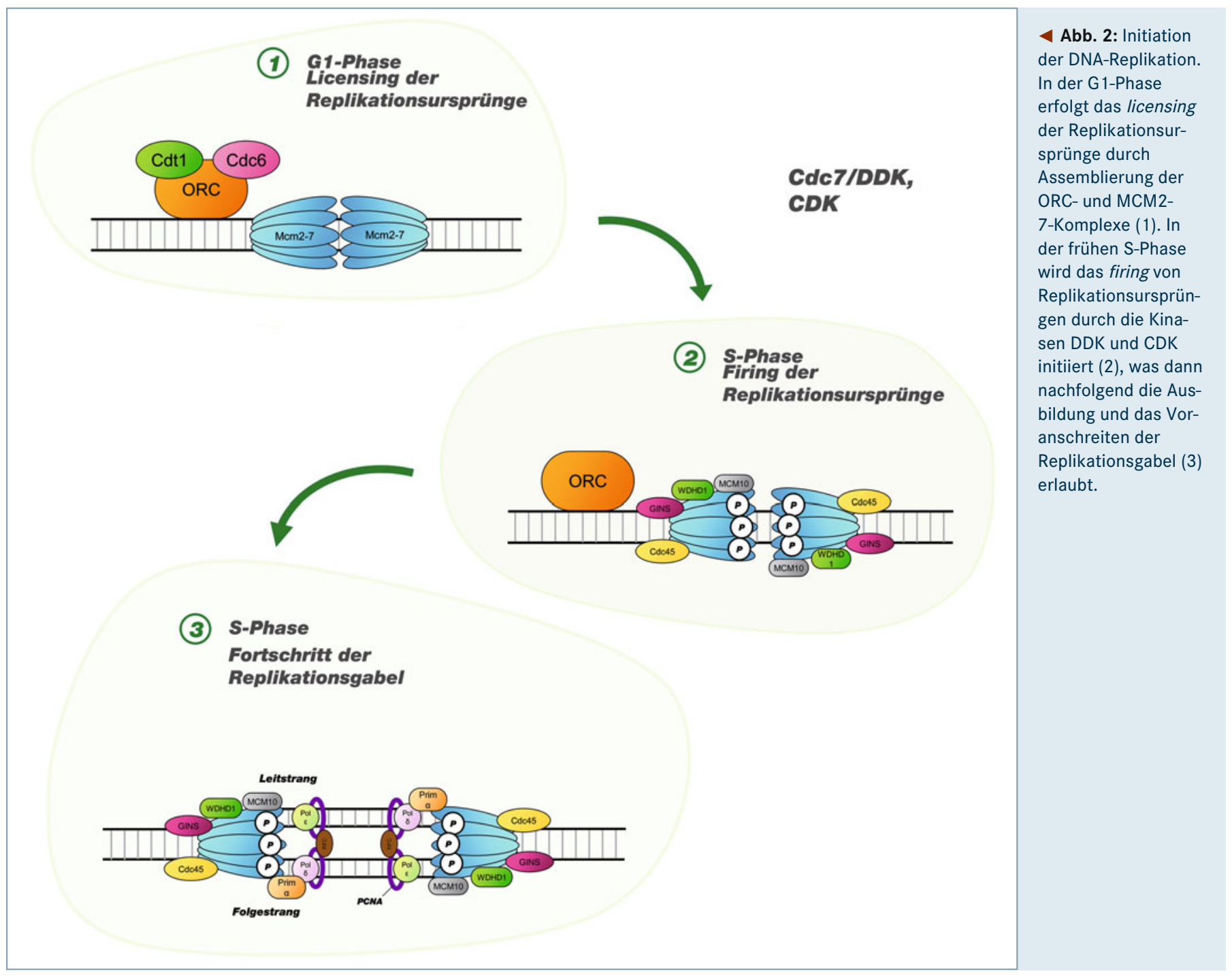

\section{Auswirkungen von Replikationsstress auf die Mitose}

Während starker Replikationsstress einen Zellzyklusarrest auslöst, kann milder Replikationsstress von der Checkpoint-Antwort unbemerkt bleiben. So können Zellen trotz partiell unvollständig replizierter DNA in die Mitose eintreten. Vor kurzem wurde gezeigt, dass in der Mitose noch die DNA-Neusynthese vervollständigt werden kann. Diese mitotische DNA-Synthese (MiDAS) beruht auf Mechanismen der break-induced repair (BIR) [14].

Ist die Reparatur durch MiDAS nicht erfolgreich, können ultra fine bridges (UFBs) die Folge sein. Es handelt sich hierbei wahrscheinlich um Bereiche unreplizierter DNA, welche die vollständige Trennung der Schwesterchromatiden in der Anaphase verhindern. Die Folge von UFBs können DNABrüche und die Ausbildung von Chromosomen-Fragmenten sein. Somit kann Replikationsstress auch in der Mitose zu S-CIN beitragen [12,13].
Vor kurzem konnte unser Labor zeigen, dass bereits eine geringe Verlangsamung der Replikation signifikante Auswirkungen in der Mitose haben kann. Neben UFBs wurden vor allem lagging chromosomes in der Anaphase detektiert. Interessanterweise induziert milder Replikationsstress unter diesen Bedingungen eine erhöhte MikrotubuliDynamik in mitotischen Spindeln, was wiederum kausal mit Chromosomen-Fehlverteilungen in Zusammenhang steht [9]. Somit scheint milder Replikationsstress, der in Krebszellen häufig detektiert wird, ein wichtiger Auslöser für eine abnormale Mikrotubuli-Dynamik und für W-CIN zu sein. Es ist daher von größtem Interesse, die funktionellen Wechselwirkungen zwischen DNA-Replikationsstress und Mitosedefekten im Detail zu untersuchen, um die Grundlage der Entstehung von chromosomaler Instabilität als treibende Kraft für die Tumorentstehung und -progression besser zu verstehen.

\section{Literatur}

[1] Burrell AR, McGranahan N, Bartek J, Swanton C (2013) The causes and consequences of genetic heterogeneity in cancer evolution. Nature 501: 338-345

[2] Bastians H (2015) Causes of chromosomal instability. Recent Results Cancer Res 200: 95-113

[3] Gregan J, Polakova S, Zhang L et al. (2011) Merotelic kinetochore attachment: causes and effects. Trends Cell Biol 21: $374-381$

[4] Ganem JN, Godinho AS, Pellman D (2009) A mechanism linking extra centrosomes to chromosomal instability. Natur 460: 278-282

[5] Ertych N, Stolz A, Stenzinger A et al. (2014) Increased microtubule assembly rates influence chromosomal instability in colorectal cancer cells. Nat Cell Biol 16: 779-791 [6] Stolz A, Ertych N, Kienitz A et al. (2010). The CHK2 BRCA1 tumour suppressor pathway ensures chromosomal stability in human somatic cells. Nat Cell Biol 12: 492-499 [7] Ertych N, Stolz A, Valerius O et al. (2016) CHK2-BRCA1 tumor-suppressor axis restrains oncogenic Aurora-A kinase to ensure proper mitotic microtubule assembly. Proc Natl Acad Sci U S A 113: 1817-1822

[8] Schmidt KA, Pudelko K, Boekenkamp EJ et al. (2020) The p53/p73 - p21(CIP1) tumor suppressor axis guards against chromosomal instability by restraining CDK1 in human cancer cells. Oncogene, doi: 10.1038/s41388-020-01524-4 [9] Böhly N, Kistner M, Bastians H (2019) Mild replication stress causes aneuploidy by deregulating microtubule dynamics in mitosis. Cell Cycle 18: 2770-2783 [10] Burrell AR, McClelland ES, Endesfelder D et al. (2013) Replication stress links structural and numerical cancer chromosomal instability. Nature 494: 492-496 
- Abb. 3: Verschiedene Faktoren können zu Replikationsstress führen. A, eine inadäquate Aktivierung der Replikationsursprünge. B, Replikation unter ungünstigen metabolischen Bedingungen. C, lokale Hindernisse der Replikationsgabel. D, Konflikte von DNAReplikation und Transkription.

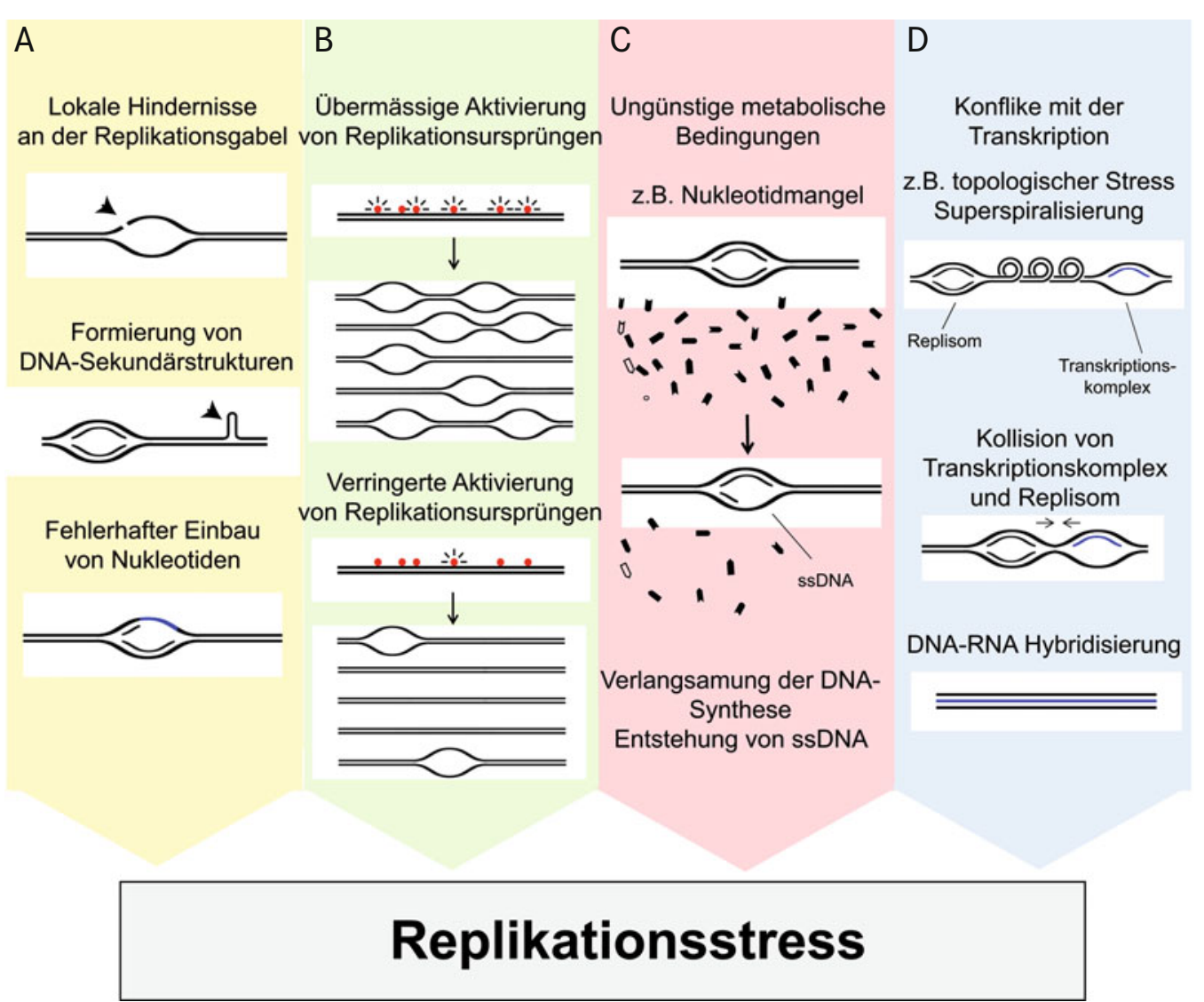

[11] Fragkos M, Ganier O, Coulombe P, Méchali M (2015) DNA replication origin activation in space and time. Nat Rev Mol Cell Biol 16: 360-374

[12] Gaillard H, Garcia-Muse T, Aguilera A (2015) Replication stress and cancer. Nat Rev Cancer 15: 276-289 [13] Zeman KM, Cimprich AK (2014) Causes and consequences of replication stress. Nat Cell Biol 16: 2-9 [14] Minocherhomji S, Ying S, Bjerregaard AV et al. (2015) Replication stress activates DNA repair synthesis in mitosis. Nature 528: 286-290

Funding note: Open Access funding enabled and organized by Projekt DEAL. Open Access: Dieser Artikel wird unter der Creative Commons Namensnennun 4.0 International Lizenz veroffentlicht, welche die Nutzung, Vervielfältigun Bearbeitung, Verbreitung und Wiedergabe in jeglichem Medium und Fo erlaubt, sofern Sie den/die ursprünglichen Autor(en) und die Quelle
ordnungsgemäß nennen, einen Link zur Creative Commons Lizenz beifügen und angeben, ob Änderungen vorgenommen wurden. Die in diesem Artikel enthaltenen Bilder und sonstiges Drittmaterial unterliegen ebenfalls der genannten Creative Commons Lizenz, sofern sich aus der Abbildungslegende nichts anderes ergibt. Sofern das betreffende Material nicht unter der genannten Creative Commons Lizenz steht und die betreffende Handlung nich nach gesetzlichen Vorschriften erlaubt ist, ist für die oben aufgeführten Weiterverwendungen des Materials die Einwilligung des jeweiligen Wer

Korrespondenzadresse:

Prof. Dr. Holger Bastians

Institut für Molekulare Onkologie

Göttinger Zentrum für

Molekulare Biowissenschaften

Universitätsmedizin Göttingen

Grisebachstraße 8

D-37077 Göttingen

holger.bastians@uni-goettingen.de

DFG-Forschergruppe 2800: „Chromosome Instability: Cross-talk of DNA replication stress and mitotic dysfunction"

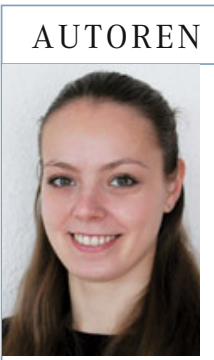

Alicia Konrath

2017-2020 Studium der Biologie (B. Sc.) an der Universität Göttingen. 2020 Bachelorarbeit am Institut für Molekulare Onkologie der Universitätsmedizin Göttingen, Göttinger Zentrum für Molekulare Biowissenschaften, im Labor von Prof. Dr. H. Bastians. Seit 2020 Studium (M. Sc.) „Molecular Life Sciences: Microbiology, Biotechnology and Biochemistry" an der Universität Göttingen.

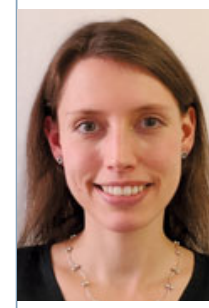

Ann-Kathrin Schmidt

2010-2013 Studium der Biochemie/Molekularbiologie (B. Sc.) an der Universität Jena. 2013-2016 Studium der Molekularen Medizin (M. Sc.) an der Universität Jena. Seit 2016 Promotion an der Universitätsmedizin Göttingen, Göttinger Zentrum für Molekulare Biowissenschaften, im Labor von Prof. Dr. H. Bastians.

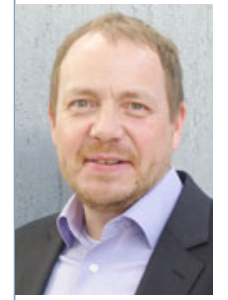

Holger Bastians

1987-1993 Studium der Biologie (Diplom) an den Universitäten Bayreuth und Osnabrück. 1993-1996 Promotion am Deutschen Krebsforschungszentrum Heidelberg. 1996-2000 Postdoktorand an der Harvard Medical School in Boston, USA, Department of Cell Biology. 2000-2010 Gruppenleiter am Institut für Molekularbiologie und Tumorforschung der Universität Marburg. Seit 2010 Professor für Zelluläre Onkologie am Institut für Molekulare Onkologie der Universitätsmedizin Göttingen, Göttinger Zentrum für Molekulare Biowissenschaften. Seit 2019 Sprecher der DFG-Forschungsgruppe 2800 Chromosome Instability: Cross-talk of DNA replication stress and mitotic dysfunction. 